\title{
Power Allocation in Massive MIMO-HWSN Based on the Water-Filling Algorithm
}

\author{
Zhe Li ${ }^{1},{ }^{1}$ Sahil Verma ${ }^{(D},{ }^{2}$ and Machao Jin $^{3}$ \\ ${ }^{1}$ School of Information Engineering, Department of Railway Policing, Railway Police College, Zhengzhou, China \\ ${ }^{2}$ Chandigarh University, Mohali, India \\ ${ }^{3}$ Department of Information and Communication, Shanxi Metallurgical Geotechnical Engineering Investigation Co., Ltd., Taiyuan, \\ Shanxi, China \\ Correspondence should be addressed to Zhe Li; lizhe@rpc.edu.cn
}

Received 30 April 2021; Revised 1 June 2021; Accepted 10 July 2021; Published 10 August 2021

Academic Editor: Xingwang Li

Copyright (C) 2021 Zhe Li et al. This is an open access article distributed under the Creative Commons Attribution License, which permits unrestricted use, distribution, and reproduction in any medium, provided the original work is properly cited.

Pilot power allocation for Internet of Things (IoT) devices in massive multi-input multioutput heterogeneous wireless sensor networks (MIMO-HWSN) is studied in this paper. The interference caused by fractional pilot reusing in adjacent cells had a negative effect on the MIMO-HWSN system performance. Reasonable power allocation for users can effectively weaken the interference. Motivated by the water-filling algorithm, we proposed a suboptimal pilot transmission power method to improve the system capacity. Simulation results show that the proposed method can significantly improve the uplink capacity of the system and explain the influence of different pilot transmission power on the performance of the system, but the complexity of the system almost does not increase.

\section{Introduction}

Due to the continued development of $5 \mathrm{G}$ and the gradual proliferation of $6 \mathrm{G}$, the wireless data traffic is estimated to reach the unprecedented quantity. Most of the future dataintensive applications handling a massive number of connected IoT will demand high data rates with low latency in some scenarios (such as vehicle network, smart city, and remote health) $[1,2]$. MIMO, millimeter wave (mmWave), nonorthogonal multiple access (NOMA), and heterogeneous cellular network (HetNet) have been broadly investigated from all aspects of academia, research, and industry at home and abroad [3-9]. Massive MIMO technology configures a large number of antennas for multiple base stations (BSs), and the transmitted signals can be dynamically adjusted horizontally and vertically, thus improving the performance of wireless communication systems. The technology was introduced by Bell Laboratories in 2010 [10-12]. Wireless sensor networks (WSNs) play a very important role in telemedicine, electronic instruments, transportation, and many other fields. WSN is equipped with abundant sensor nodes (SN) which can sense the external environment and perform communication and calculation and connect extensive wireless devices through the wireless systems [13-19]. HWSN usually consists of low power consumption and a tiny village network stack on the macrocell, though the space and spectrum reuse technology upgrades the capacity of the hot spots of the overlay network [20-22]. In this paper, the massive MIMO-HWSN system embodies some small-cell base stations (SBSs) and macrocell base stations (MBSs).

In actual wireless communication systems, the number of orthogonal pilots is often less than active users, so it is impossible for each user to use the orthogonal pilots because of the limited length of the coherence time. When several users in the system use the same pilots, pilot contamination will result in inaccurate channel estimation and the BS cannot obtain accurate channel state information (CSI) [23-29]. In the time-division duplex (TDD) massive MIMO-HWSN system which employs pilots to conduct uplink channel estimation, the pilot contamination seriously hinders the increasing 
trend of wireless communication system capacity and delays the process of standardization and industrialization $[30,31]$.

In the current research, there are many means to mitigate the pilot contamination. In terms of channel estimation, some researchers designed new channel estimation methods or improved the existing channel estimation algorithm according to the characteristics of the system to reduce channel estimation errors and directly alleviate the pilot contamination. In addition to the classical Minimum Mean Square Error (MMSE) and Least Square (LS) channel estimation [32], a variety of channel estimation methods that may become the industry standard of massive MIMO systems has also been proposed. Reference [33] proposes a channel estimation method based on mutual cooperation between base stations (BSs) in the system. This scheme uses additional second-order statistical information of channels to distinguish users. Reference [34] proposes a blind channel estimation method based on subspace projection without mutual cooperation between cells. This blind channel estimation scheme is combined with an appropriate power allocation scheme which can effectively alleviate the pilot contamination in massive MIMO systems. Reference [35] gives a channel estimation method which has no special requirements on the encoding and signal structure of the transmitting end. The channel response vectors corresponding to different users will be close to orthogonality when the number of BS antennas is close to infinity. This scheme uses the feature of the massive MIMO system to decompose the covariance matrix of the received signal by eigenvalue decomposition, so as to achieve the purpose of distinguishing users. References [31, 32, 36-39] gave a channel estimation scheme combined with geographic location information. After the channel estimation based on the uplink pilot sequence, the scheme performs a postprocessing process based on the fast Fourier transform on the steering vector at the BS. Hence, the users with different angles of arrival (AOAs) can be effectively distinguished.

For the pilot allocation, an allocation scheme based on channel covariance matrix is proposed in $[36,37]$. References [38-40] proposed a partial pilot sequence (frequency) multiplexing scheme or soft multiplexing scheme. Reference [41] modeled the pilot scheduling problem as an optimal permutation and combination problem and obtained the corresponding low-complexity approximate solution. Reference [42] uses the unique sparse characteristics of broadband system channels in the time domain to distinguish between target users and interfering users and designs a pilot sequence allocation scheme that can randomize pilot pollution in multiple consecutive uplink frames. For mitigating the interference between cells and increase the system capacity in [43], a pilot allocation scheme with cell sectorization is given which ensures that the pilot sequence used in each sector and adjacent cells are orthogonal. Reference [44] grouped cell edge users based on channel AOA information, thus effectively mitigating the adverse effects of interfering users on target users. In [45], the Rice fading channel model is adopted, and the pilot sequence is allocated according to the line-of-sight interference between different users. In [46], a problem is formulated to jointly optimize the number of BS antennas, power allocation and guide neck sequence allocation are constructed, and a step-by-step solution is proposed.

In this paper, a macrocell SN (MSN) uplink pilot transmit power allocation scheme based on location-aware channel estimation and a novel pilot allocation method is proposed and obtains the effects of different power allocations on improving massive MIMO-HWSN system performance. Inspired by the main idea of power allocation in the waterfilling algorithm, that is to say, when MSN has better channel gain, more transmit power will be allocated, we apply the power allocation method according to the constraint conditions and only implement this method for MSNs. In the simulation, the results of pilot power average transmission are compared, which shows that the proposed method can effectively improve the performance of the system.

The remainder of this paper is organized as follows. The massive MIMO-HWSN system and the channel model are presented in Section II. A novel channel estimation method is described in Section III. Section IV proposes a pilot power allocation scheme. The simulation results under different conditions are given in Section V. Finally, we summarize this paper in Section VI.

1.1. Notations. The upper case and lower case boldface represent matrices and vectors, respectively. $(\mathbf{A})^{T}$ and $(\mathbf{A})^{H}$ denote the transpose and conjugate transpose of $\mathbf{A}$, respectively. $\mathbb{C}$ denotes the complex number. $\mathbb{E}\{\mathbf{B}\}$ and $\operatorname{Tr}(\mathbf{B})$ indicate the statistical expectation and the trace of $\mathbf{B}$ for random variables $\mathbf{B}$.

\section{System Model}

We consider a heterogeneous multicell multiuser massive MIMO system with $C$ hexagonal macrocells. As shown in Figure 1, there are $P$ covered small cells in each macrocell. At the coherent time, one single-antenna SSN is served in each small cell and $K_{m}$ single-antenna MSNs in a macrocell. The number of SSNs is far less than that of the MSNs in each cell of this system. The MBS has $M$ antennas in each cell, where $M>>K_{m}$, while the SBS has one antenna.

The time division duplex (TDD) model is applied in the system to reduce the large overhead of training in the MIMO-HWSN system, which is shown in Figure 2. The response vectors of the uplink channel change with time but remain unchanged in the coherent time.

At the uplink training, all MSNs and SSNs from their own cells send the pilot signal to corresponding BSs. $\phi_{k}=\left[\phi_{k, 1}, \phi_{k, 2} \cdots, \phi_{k, \tau}\right] \in \mathbb{C}^{1 \times \tau}$ is the pilot sequence of the $k$-th MSN of a cell, $k \in\left[1, K_{m}\right]$, and $E\left\{\phi_{k} \phi_{k}^{H}\right\}=1 . \tau$ is denoted as the length of the pilot sequence. The pilot sequences are orthogonal when $k$ has different values, i.e., $\phi_{k_{1}} \phi_{k_{2}}^{H}=0$, where $k_{1} \neq k_{2}$ and $k_{1}, k_{2} \in\left[1, K_{m}\right]$. In the MIMO-HWSN system, the $k$-th SSN reuses the pilot sequences of the $s_{k}$-th MSN in the same cell, which is denoted as $\phi_{s_{k}} \in\left\{\phi_{1}, \phi_{2}, \cdots \phi_{K_{m}}\right\}$, where $s_{k} \in\left\{1,2, \cdots, K_{m}\right\}$ and $k \in[1, P]$. 


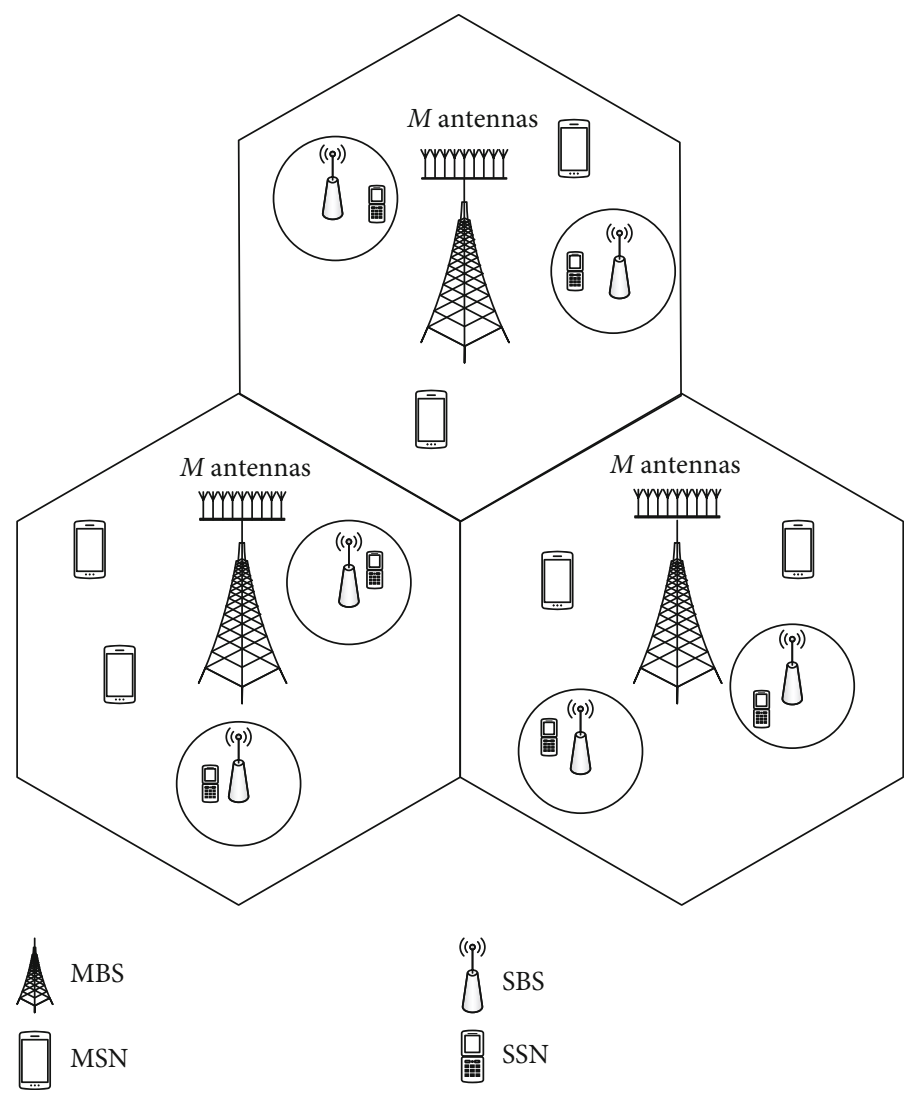

Figure 1: The MIMO-HWSN system model with $C=3$ and $P=2$.

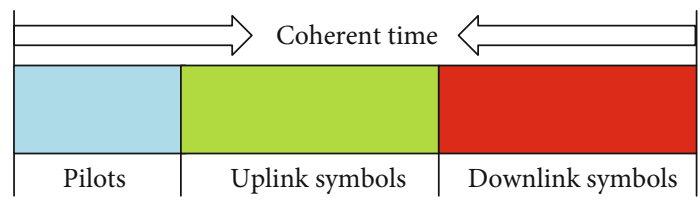

FIgURE 2: Coherent time model.

We consider the narrow-band channel which is a commonly used channel model, which can be modeled as

$$
\mathbf{h}_{b, c, k}=\frac{1}{\sqrt{Q}} \sum_{q=1}^{Q} \mathbf{v}\left(\theta_{b, c, k, q}\right) \omega_{b, c, k, q}
$$

where $\omega_{b, c, k, q}$ stands for the large-scale fading parameter of the $i$-th path, which contains the shadow fading and path-loss; $Q$ denotes the path of the $k$-th MSN in the $c$-th cell to the $b$-th MBS; $\mathbf{v}(\theta)$ indicates the steering vector; and $\theta_{b, c, k, q}$ denotes the AOA of the $q$-th path. The AOA is small and can be assumed in $\left[\theta_{\min }, \theta_{\max }\right]$ generally. The steering vector is given in this expression for uniformly spaced linear array as

$$
\mathbf{v}(\theta)=\left[\begin{array}{c}
1 \\
e^{-j 2 \pi \frac{D}{\lambda} \cos (\theta)} \\
\cdots \\
e^{-j 2 \pi \frac{(M-1) D}{\lambda}} \cos (\theta)
\end{array}\right],
$$

where $\lambda$ denotes the wavelength, $D$ indicates the antenna spacing at MBS, and $D \leq \lambda / 2$. The received signal by the MBS in the $c$-th cell can be denoted as

$$
\mathbf{Y}_{b}=\sum_{c=1}^{C} \sum_{k=1}^{K_{m}} \sqrt{p_{c, k}} \mathbf{h}_{b, c, k} \phi_{k}+\sum_{c=1}^{C} \sum_{p=1}^{P} \sqrt{p^{\prime}{ }_{c, k}} \mathbf{h}_{b, c, k}^{\prime} \phi_{s_{k}}+\mathbf{N}_{b},
$$

where $\mathbf{Y}_{b} \in \mathbb{C}^{M \times \tau}$ denotes the received signal matrix and $\mathbf{N}_{b} \in \mathbb{C}^{M \times \tau}$ indicates the additive white Gaussian noise (AWGN) matrix; $p_{c, k_{m}}$ and $p_{c, k_{s}}^{\prime}$ are pilot transmitted power of the $k_{m}$-th MSN and $k_{s}$-th SSN in the $c$-th cell to the $b$-th MBS, respectively; and $\mathbf{h}_{b, c, k_{m}}=\left[h_{b, c, k_{m}, 1} h_{b, c, k_{m}, 2} \cdots h_{b, c, k_{m}, M}\right]^{T}$ and $\mathbf{h}_{b, c, k_{s}}=\left[h_{b, c, k_{s}, 1} h_{b, c, k_{s}, 2} \cdots h_{b, c, k_{s}, M}^{\prime}\right]^{T}$ denote channels with $h_{b, c, k_{m}, q}$ and $h_{b, c, k_{s}, q}$ which denote the channel coefficient between the $k_{m}$-th MSN and $k_{s}$-th SSN in the $c$-th cell and the $b$-th antenna of the $b$-th MBS, respectively. The orthogonal pilot sequence is used for the LS channel estimation at the MBS. That is, right multiplying $\mathbf{Y}_{b}$ by $\phi_{k}^{H}$ can be written as

$$
\begin{aligned}
& \widehat{\mathbf{h}}_{b, b, k}=\frac{\mathbf{Y}_{b} \boldsymbol{\xi}_{k}^{H}}{\sqrt{p_{b, k}}}=\mathbf{h}_{b, b, k}+\sum_{c=1, c \neq b}^{C} \sqrt{\frac{p_{c, k}}{p_{b, k}}} \mathbf{h}_{b, c, k}
\end{aligned}
$$

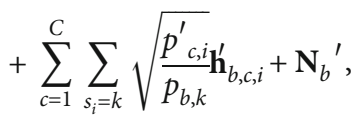


where $\mathbf{N}_{b}{ }^{\prime}=\mathbf{N}_{b} \phi_{k}^{H} / \sqrt{p_{b, k}}$ indicates the equivalent noise matrix for channel estimation. Obviously, for the $k$-th MSN in the $b$-th cell, the part containing the $\mathbf{h}_{b, c, i}$ denotes the intertier interference from the SSNs using the same pilot as the MSN, while the part that contains $\mathbf{h}_{b, c, k}$ is the intercell interference casued by MSNs in the adjacent cell.

The received signal by the $i$-th SBS in the $b$-th macrocell is written as

$\mathbf{y}_{b, i}^{\prime}=\sum_{c=1}^{C} \sum_{k=1}^{K_{m}} \sqrt{\xi_{b, i, c, k}} g_{b, i, c, k} \phi_{k}^{T}+\sum_{c=1}^{C} \sum_{k=1}^{P} \sqrt{{ }^{\prime} \xi_{b, i, c, k}}{ }^{\prime} g_{b, i, c, k} \phi_{s_{k}}^{T}+\gamma_{b, i}$,

where $\xi_{b, i, c, k_{m}}$ and ' $\xi_{b, i, c, k_{s}}$ are pilot transmitted powers of the $k_{m}$-th MSN and $k_{s}$-th SSN, respectively; $g_{b, i, c, k}$ and ' $g_{b, i, c, k}$ are the channel coefficients linking the $k_{m}$-th MSN and $k_{s}$ -th SSN of the $c$-th cell to the $i$-th SBS of the $b$-th macrocell, respectively; and $\gamma_{b, i}$ denotes the AWGN vector. Similarly, we can get the channel estimation $\hat{g}_{b, i, b i}$ which can be denoted as

$$
\begin{aligned}
\hat{g}_{b, i, b, i}= & \frac{\phi_{s_{i}}^{H} \mathbf{y}_{b, i}^{\prime}}{\sqrt{\xi_{b, i, b, i}}}=\prime^{\prime} g_{b, i, b, i}+\underbrace{\sum_{j=1}^{C} \frac{g_{b, i, c, s_{i}}}{\sqrt{\xi_{b, i, b, i}}}}_{\mathrm{P} 1} \\
& +\underbrace{\sum_{s_{k}=s_{i}, k \neq i} \frac{\stackrel{\prime}{g}_{b, i, b, k}}{\sqrt{\xi_{b, i, b, i}}}}_{\mathrm{P} 2}+\underbrace{\sum_{j=1, j \neq l}^{C} \sum_{s_{k}=s_{i}} \frac{\stackrel{g}{g}_{b, i, c, k}}{\sqrt{\xi_{b, i, b, i}}}}_{\mathrm{P} 3}+\zeta_{b, i},
\end{aligned}
$$

where $\zeta_{b, i}=\phi_{s_{i}}^{H} \gamma_{b, i} / \sqrt{\xi_{b, i, b, i}}$ denotes the equivalent noise at the channel estimate. Behind the second equal sign of (4), P1 is the intertier interference caused by the $s_{i}$-th MSN of each macrocell. The sum of $\mathrm{P} 2$ and $\mathrm{P} 3$ denotes the intercell interference which is caused by other SSNs with the same pilot. In this formula, P2 is the intercell interference casued by the SSNs in the same macrocell, and $\mathrm{P} 3$ can be denoted as the intercell interference from SSNs in the adjacent macrocell.

Based on the analysis of the above equations, the fundamental cause of pilot contamination is that different MSNs in the system reuse the same pilot sequence. The length of the coherent time in a massive MIMO system is limited, which is shown in Figure 2, and this character limits the length of the pilot sequence. The limited length of pilot sequences also means that the number of orthogonal pilot sequences available in the system is limited. On the other hand, the number of users is generally huge in a massive MIMO system, so it is impossible for all users to allocate mutually orthogonal pilot sequences. In this case, the phenomenon of pilot multiplexing occurs. Moreover, the base station in each cell will not only receive the uplink pilot sequences sent by SNs in the cell but also receive the pilot sequences sent by SNs in other cells. Furthermore, when the channel estimation is conducted at the BS, SNs in other cells using the same pilot sequence will be affected, resulting in inaccurate channel estimation results. Specifically in this system model, the $k$-th $\mathrm{SN}\left(k=1,2,3, \cdots, K_{m}\right)$ in each cell reuses the same pilots. The base station in the $b$-th cell simultaneously receives uplink pilot sequences from SNs in other cells. So the channel estimation result obtained by the BS based on the received pilot signal includes both the channel response parameters of users in the $b$-th cell and the channel response parameters from users in other cells.

We only consider the process of MSNs to its corresponding MBSs in the stage of transmitting data. Actually, the stages of SSNs are very similar to those of MSNs. The received signal $\mathbf{y}_{b} \in \mathbb{C}^{M \times 1}$ in $b$-th can be denoted as

$$
\mathbf{y}_{b}=\sqrt{p_{m}} \sum_{c=1}^{C} \sum_{k=1}^{K_{m}} \mathbf{h}_{b, c, k} x_{b, c, k}+\sqrt{p_{s}} \sum_{c=1}^{C} \sum_{k=1}^{P} \mathbf{h}_{b, c, k}^{\prime} \dot{x}_{b, c, k}+\varepsilon_{b},
$$

where $x_{b, c, k_{m}}$ and $\dot{x}_{b, c, k_{s}}$ denote the symbols transmitted by the $k_{m}$-th MSN and $k_{s}$-th SSN in the $c$-th cell to $b$-th MBS, respectively; $E\left\{\left|x_{b, c, k}\right|^{2}\right\}=E\left\{\left|\dot{x}_{b, c, k}\right|^{2}\right\}=1$; and $p_{m}$ and $p_{s}$ are the data transmit power which are assumed to be identical for all MSNs and SSNs, respectively. $\varepsilon_{b} \in \mathbb{C}^{M \times 1}$ denotes the AWGN vector. The MBS demodulates the data according to the channel estimation $\widehat{\mathbf{h}}_{l, l, k}$, which is obtained in the previous stage. In this stage, we consider the matched-filter (MF) detector, and the detected signal from the $k$-th MSN to $l$-th MBS in the $j$-th cell can be modeled as

$$
\begin{aligned}
\widehat{\mathbf{x}}_{b, b, k}= & \frac{\left(\mathbf{h} \wedge_{b, b, k}\right)^{H} \mathbf{y}_{b}}{\sqrt{p_{m}}}=\underbrace{\sum_{c=1}^{C} \sum_{k=1}^{K_{m}}\left(\mathbf{h} \wedge_{b, b, k}\right)^{H} \mathbf{h}_{b, c, k} x_{b, c, k}}_{\mathrm{S} 1} \\
& +\underbrace{\sqrt{\frac{p_{s}}{p_{m}}} \sum_{j=1}^{C} \sum_{k=1}^{P}\left(\mathbf{h} \wedge_{b, b, k}\right)^{H} \mathbf{h}_{b, c, k} \dot{x}_{b, c, k}}_{\mathrm{S} 2}+\underbrace{\frac{\left(\mathbf{h} \wedge_{b, b, k}\right)^{H} \varepsilon_{b}}{\sqrt{p_{m}}}}_{\mathrm{S} 3},
\end{aligned}
$$

where $\mathrm{S} 1$ can be expressed as

$$
\begin{aligned}
& \mathbf{S} 1=\left(\mathbf{h} \wedge_{b, b, k}\right)^{H} \mathbf{h}_{b, b, k} x_{b, b, k}+\underbrace{\sum_{k^{\prime} \neq k}^{K_{m}}\left(\mathbf{h} \wedge_{b, b, k}\right)^{H} \mathbf{h}_{b, b, k^{\prime}} x_{b, b, k^{\prime}}}_{\text {S1a }} \\
& +\underbrace{\sum_{c^{\prime} \neq c}^{C} \sum_{k=1}^{K_{m}}\left(\mathbf{h} \wedge_{b, b, k}\right)^{H} \mathbf{h}_{b, c}{ }^{\prime, k} x_{b, c}{ }^{\prime \prime, k}}_{\text {Slb }},
\end{aligned}
$$

where S1a represents the interference from MSNs of the same macrocell and S2a denotes the interference from MSNs of the different macrocells. The SINR of the $k$-th MSN in the $b$-th macrocell is computed by (10). A channel estimation method will be introduced to get the more accurate $\widehat{\mathbf{h}}$. 


\section{A Novel Location-Aware Channel Estimation Method}

$$
\operatorname{SINR}_{c, k}=\frac{\left|\left(h \wedge_{b, b, k}\right)^{H} h_{b, b, k}\right|^{2}}{\left.\sum_{k^{\prime} \neq k}^{K_{m}}\left|\left(h \wedge_{b, b, k}\right)^{H} h_{b, b, k}\right|^{\prime}\right|^{2}+\sum_{c^{\prime} \neq c}^{C} \sum_{k=1}^{K_{m}}\left|\left(h \wedge_{b, b, k}\right)^{H} h_{b, c^{\prime}, k}\right|^{2}+\left(p_{s} / p_{m}\right) \sum_{c=1}^{C} \sum_{k=1}^{P}\left|\left(h \wedge_{b, b, k}\right)^{H} h_{b, c, k}^{\prime}\right|^{2}+\left|\left(h \wedge_{b, b, k}\right)^{H} \varepsilon_{b}\right|^{2} / p_{m}} .
$$

$\widehat{\mathbf{h}}$ can be rewritten as

$$
\widehat{\mathbf{h}}=\mathbf{h}+\mathbf{h}_{I}+\mathbf{n},
$$

where $\mathbf{h}_{I}$ denotes the sum of all interfering channels, $\mathbf{h}$ is the profitable channel vector, and $\mathbf{n}$ is the AWGN vector. Actually, we regard (2) as a single frequency signal, and its frequency is $f_{a}=(D / \lambda) \cos (\theta)$ when $M \longrightarrow \infty$, and we denote $\mathbf{F}$ as the $N$-point Fast Fourier Transform (FFT) of $\widehat{\mathbf{h}}$. Then, the $N$-point DFT of $\mathbf{a}(\theta)$ can be written as

$$
X(k)=\sum_{m=1}^{M-1} a(m) \exp \left(-j \frac{2 \pi}{N} k m\right),
$$

where $a(m)=\exp (-j 2 \pi(m D / \lambda) \cos (\theta))$, which is the $m$-th element of $a(\theta)$, and $m \in[0, M-1], k \in[0, N-1]$, $\max [X(k)]=M$, let $k_{\lim }=\operatorname{argmax}|X(k)|, k \in[0, N-1], k_{\lim }$ $=\left\lfloor g_{N}(\theta)\right\rceil$, where $\left\lfloor g_{N}(\theta)\right\rceil$ represents the integer rounding for $g_{N}$, and it can be expressed as

$$
g_{N}(\theta)= \begin{cases}N-N \frac{D}{\lambda} \cos (\theta), & \theta \in\left[0, \frac{\pi}{2}\right) \\ -N \frac{D}{\lambda} \cos (\theta), & \theta \in\left[\frac{\pi}{2}, \pi\right] .\end{cases}
$$

The large frequency domain values of $\mathbf{F}$ should be in the region $J\left(k_{\min }, k_{\max }\right)$ when $X(k)$ approachs to the maximal value $M$, which can be written as

$$
J\left(k_{\min }, k_{\max }\right)= \begin{cases}{\left[0, k_{\max }\right] \cup\left[k_{\min }, N\right],} & \theta_{\min } \in\left[0, \frac{\pi}{2}\right] \\ & \theta_{\text {max }} \in\left[\frac{\pi}{2}, \pi\right] \\ {\left[k_{\min }, k_{\max }\right],} & \text { otherwise }\end{cases}
$$

where $k_{\min }=\left\lfloor g_{N}\left(\theta_{\min }\right)\right\rceil$ and $k_{\max }=\left\lfloor g_{N}\left(\theta_{\max }\right)\right\rceil$. The values of $\mathbf{F}$ outside the interval $J\left(k_{\min }, k_{\max }\right)$ are set to zero, which is equivalent to canceling the signals outside the interval $\left[\theta_{\min }, \theta_{\max }\right]$, leading to the interference and the effect of the noise reducing. Then, the Inverse Fast Fourier Transform (IFFT) of $\mathbf{E}$ can be expressed as

$$
\mathbf{f}=[f \wedge(0) f \wedge(1) \cdots f \wedge(N-1)]^{T},
$$

The estimation of the channel coefficient vector can be written as

$$
\overline{\mathbf{h}}=[f \wedge(0) f \wedge(1) \cdots f \wedge(M-1)]^{T} .
$$

\section{Uplink Pilot Power Allocation Based on Water-Filling Algorithm}

First, we introduce a pilot assignment method based location for massive MIMO-HWSN system. The novel channel estimation at the MBSs shown in the previous section is utilized, and some orthogonal pilot sequences could be reused by SSNs so that the idea can support more users simultaneously reducing the interference partly.

In Figure 3, we define $d_{n, k, j}$ as the Euclidean distance between the $j$-th macrocell and the $n$-th interfering user. $\mathbf{z}(\theta)=[\cos (\theta) \sin (\theta)]^{T}$ indicates the directional vector with unit length. In the $j$-th macrocell, there is a function to measure the interference at the MBS from the $k$-th MSN to the $n$-th interfering user, which is written as

$$
I_{n, k, c}=\frac{\left|\mathbf{z}^{T}\left(\theta_{n, k, n}\right) \mathbf{z}\left(\theta_{n, k, j}\right)\right|}{d_{n, k, c}^{\gamma}},
$$

where $\theta_{n, k, j}$ denotes the AOA of the $k$-th MSN in the $j$-th macrocell at the $n$-th MBS, and we denote $\gamma$ as the pathloss exponent. Then, the interference to all MSNs can be expressed as

$$
\mathbf{T}_{\mathrm{MSN}}=\sum_{b=1}^{C} \sum_{k=1}^{K_{m}} \sum_{n=1, n \neq l}^{C} \mathbf{I}_{n, k, c}+\sum_{l=1}^{C} \sum_{k=1}^{K_{m}} \sum_{s_{i}=k} \mathbf{I}_{i, k, c}
$$

where $\mathbf{T}_{\mathrm{MSN}}$ is the interference from MSNs and the second part of $\mathbf{T}_{\text {MSN }}$ is the interference from SSNs.

The assignment not applicable to this scenario that the SBSs with only one antenna. Thus, the interference at the SBS is

$$
I_{n, k, c}^{\prime}=\frac{1}{d_{n, k, c}^{\gamma}},
$$

where $I_{n, k, j}^{\prime}$ denotes the interference from the $k$-th SSN to the $n$-th interfering user in the $j$-th macrocell. Similarly, the interference to all SSNs can be written as 


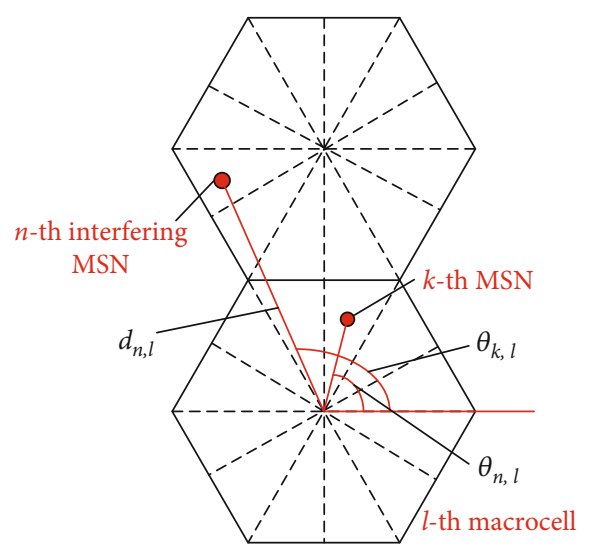

FIGURE 3: Illustration of $k$-th MSN in $l$-th macrocell with respect to $n$-th interfering MSN.

$$
\mathbf{T}_{\mathrm{SSN}}=\sum_{b=1}^{C} \sum_{k=1}^{P} \sum_{n=1}^{C} \mathbf{I}_{n, k, c}^{\prime}+\sum_{b=1}^{C} \sum_{k=1}^{P} \sum_{s_{i}=s_{k}, i \neq k} \mathbf{I}_{i, b, k}^{\prime} .
$$

The first part of $\mathbf{T}_{\mathrm{SSN}}$ is the interference from MSNs, and the second part of $\mathbf{T}_{\mathrm{SSN}}$ is the interference from SSNs. All interference in this system can be modeled as $\mathbf{T}=\mathbf{T}_{\mathrm{MSN}}+\mathbf{T}_{\mathrm{SSN}}$.

The traditional optimal mode can find the optimal pilot allocation method with the minimum $T$ value by exhaustively calculating various schemes, but for MIMO-HWSN, this is impossible because its complexity is very high.

In this model, there is a hexagonal macrocell, which is evenly divided into $K_{m}$ sectors, and there is only one MSN in each sector. We determine the number of MSN $K_{m}$ supported by each macrocell, but we are not sure where the MSN is located in each sector. Then, we introduce a pilot allocation algorithm to get a suboptimal pilot allocation scheme, which is divided into four steps:

(1) The same pilot will be assigned to the two sectors which have the least interference between two sectors in an adjacent macrocell

(2) In the central macrocell, we assign the pilots to each SSN. Since different pilot allocation modes lead to different interferences, the pilot allocation mode can be determined after finding the minimize intertier interference

(3) We assign the pilots to SSNs in each macrocell and the remaining unallocated sectors through exhaustive search, which depends on ensuring the minimize interference metric I. When calculating interference from MSNs, only the SSNs in the same macrocell with the MSNs are considered

(4) Checking for the interference between SSNs and assigning same pilots. A new pilot allocation method is proposed to handle the serious interference

After the four-step pilot assignment algorithm, a suboptimal pilot allocation model with reasonable computational complexity can be determined.
After determining the pilot allocation, the mode of allocating the total pilot transmission power to the MSNs will significantly affect the system capacity. The uplink capacity of the $k$-th MSN is given by

$$
C_{c, k}=E\left\{\log _{2}\left(1+\operatorname{SINR}_{c, k}\right)\right\}
$$

The uplink SINR $_{j, k}$ of the $k$-th MSN in $j$-th macrocell can be obtained through (10). In order to achieve a larger average achievable system capacity, the optimization problem can be written as

$$
\begin{array}{ll}
\max _{\{\Delta\}} & \sum_{c=1}^{C} \sum_{k=1}^{K_{m}} \operatorname{SINR}_{c, k}, \\
\text { s.t. } & \sum_{c=1}^{C} \sum_{k=1}^{K_{m}} p_{c, k}=p_{\text {total }},
\end{array}
$$

where $\{\Delta\}$ represents the optimal pilot power allocation schemes. Since it is difficult to get a closed form solution to the optimal problem, we consider proposing a suboptimal power allocation method combined with the traditional water-filling algorithm. The traditional water-filling algorithm allocates the transmit power adaptively according to channel conditions. In general, more power is allocated when the channel condition is good, and less power is allocated when the channel condition is poor, so as to maximize the SINR and transmission rate. Inspired by the water-filling algorithm, we can propose a suboptimal pilot transmit power allocation scheme on the basis of keeping the total power unchanged.

Figure 4 shows the distribution process of the water-filling algorithm. The interference energy is expressed by the reciprocal of the $\Gamma / \mathrm{SINR}_{n}$, where $\Gamma$ represents the difference of the values of SINR. Obviously, the subchannel with a large SINR has a small reciprocal $\Gamma / \mathrm{SINR}_{n}$ in Figure 4 and, thus, can allocate more energy. Otherwise, it can be allocated less energy. The SINR is too small for channel 3 , that is, $\Gamma / \mathrm{SINR}_{3}$ is too large, so no energy is allocated to this subchannel.

The result of the "water-filling algorithm" shows that the characteristic subchannel with a large channel gain is allocated with larger power to make its capacity larger; on the contrary, the characteristic subchannel with a small channel gain is allocated with smaller power to maintain a certain capacity to ensure that the characteristic subchannel with good channel conditions can transmit more information. When the conditions of a subchannel are poor to a certain extent, the transmit power may not be allocated for that channel. At this point, the subchannel does not send any data. The water-filling algorithm makes full use of the good channel conditions, discards the poor channels, and avoids the system using most of the transmit power to make up for the poor channel conditions.

Sufficient power is allocated for each WSN to guarantee the successful transmission of pilot sequences. However, when the allocated pilot transmission power is too large, it will affect the system performance of other users, so this 


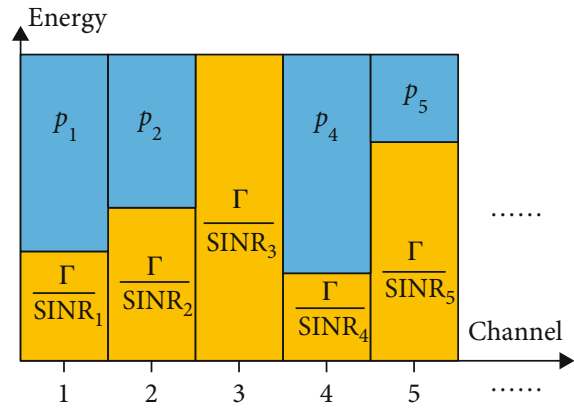

FIGURE 4: Water-filling algorithm of AWGN channel.

condition $p_{c, k} \in\left[p_{\min }, p_{\max }\right]$ should be satisfied. The values are $p_{\text {min }}=p_{\text {total }} / 2 K_{m}$ and $p_{\max }=3 p_{\text {total }} / 2 K_{m}$.

We can set the number of all MSNs to $U=L \cdot K_{m}$, and the pilot transmission power of MSNs can be written as $P_{l}=\left\{p_{1}, p_{2}, \cdots, p_{U}\right\}$, where $p_{i}=0, i \in 1,2, \cdots, U$. Then, we use the pilot to distribute the transmission power based on the water-filling algorithm, which can be described as follows:

First, the lowest power $p_{\min }$ is assigned to MSNs; that is, for all the elements $p_{i}=p_{\min }, i \in 1,2, \cdots, U$, the power not allocated is

$$
p_{\text {rest }}=p_{\text {total }}-U \cdot p_{\text {min }} .
$$

Second, a set of numbers as $T=\left\{t_{1}, t_{2}, \cdots, t_{U}\right\}$ is generated to satisfy both uniform distribution in $\left[p_{\min }, p_{\max }\right]$ and the constraint

$$
\sum_{u=1}^{U} t_{u}=p_{\text {rest }}
$$

where $t_{1}>t_{2}>\cdots>t_{U}$. Through the previous calculation, we can clearly obtain the channel gain of MSNs, marked as $H_{l}=\left\{\left|h_{1}\right|^{2},\left|h_{2}\right|^{2}, \cdots,\left|h_{U}\right|^{2}\right\}$. The remaining power is allocated to MSNs according to the order of channel gain. $\left|h_{j}\right|^{2}$ indicates the channel gain of $j$-th MSN, and the order of values in $H_{l}$ is $j^{\#}$. The pilot power of $j$-th MSN can be given as

$$
p_{j}=p_{\text {min }}+t_{j^{*}} \cdot
$$

where $t_{j^{\#}}$ is the $j^{\#}$-th value of $T$.

We can improve the system capacity by the above scheme, while satisfying the constraints in (22).

\section{Simulation Result}

The performance of our proposed scheme is evaluated via simulations. We propose a multicell multiuser MIMOHWSN system with $C=7$ macrocells, which is divided into $S=12$ sectors evenly. The number of MSNs is set as $K_{m}=$ 12 , and $P=4$ SSNs of the four reused partial orthogonal pilots are randomly distributed. The radio of the macrocell is set as $500 \mathrm{~m}$, the number of paths in the multipath chan-
TABle 1: Parameters of the simulation system.

\begin{tabular}{lc}
\hline Macrocell radius & $500 \mathrm{~m}$ \\
Number of orthogonal pilots & 12 \\
Path loss exponent & 3.5 \\
Number of paths per user $Q$ & 50 \\
Angle spread & 10 degrees \\
Variance of shadow fading & $8 \mathrm{~dB}$ \\
Transmit power at SSNs & $10 \mathrm{~mW}$ \\
Antenna spacing $D$ & $\lambda / 2$ \\
\hline
\end{tabular}

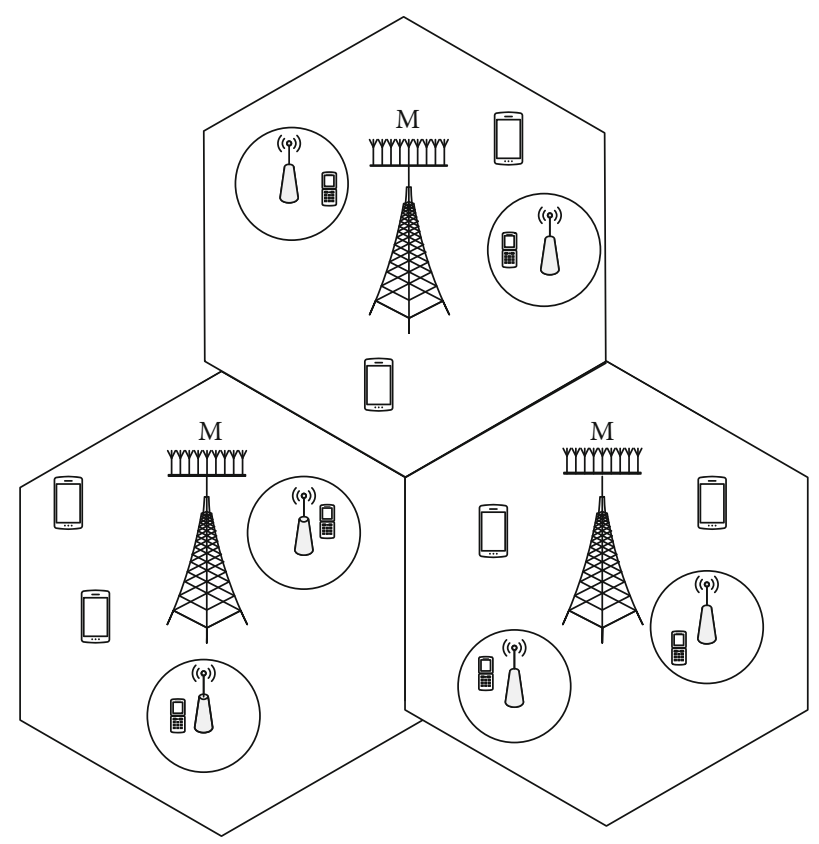

Figure 5: The model obtained after assigning the pilots.

nel is 50 , and the path loss exponent is 3.5. It is assumed that there are 12 orthogonal pilots and the angle spread is 10 degrees. The transmit power of SSNs is $0 \mathrm{dBm}$, and the variance of shadow fading is $8 \mathrm{~dB}$. The wavelength $\lambda=2 D$. All of the parameters which are used in simulations are listed in Table 1.

In the location-aware channel estimation, we set $N=$ 8192 as the size of FFT. Figure 5 is the model of a massive MIMO-HWSN system using pilot assignment based on location information. In this figure, the red dots represent the randomly distributed small cells, the red numbers indicate the pilot reused by SSN in that small cell, and the black numbers in each sector indicate the pilots to which the sector is assigned. In the allocation process, we ensure that the interference between MSNs using the same pilot is always minimal. In this model, the pilots used by MSNs in each sector of the central macrocell are randomly assigned. The pilot allocation in the central macrocell will affect the pilot types used by other SNs in the system. We assume that the pilot settings in the central macrocell are as shown in the figure. 


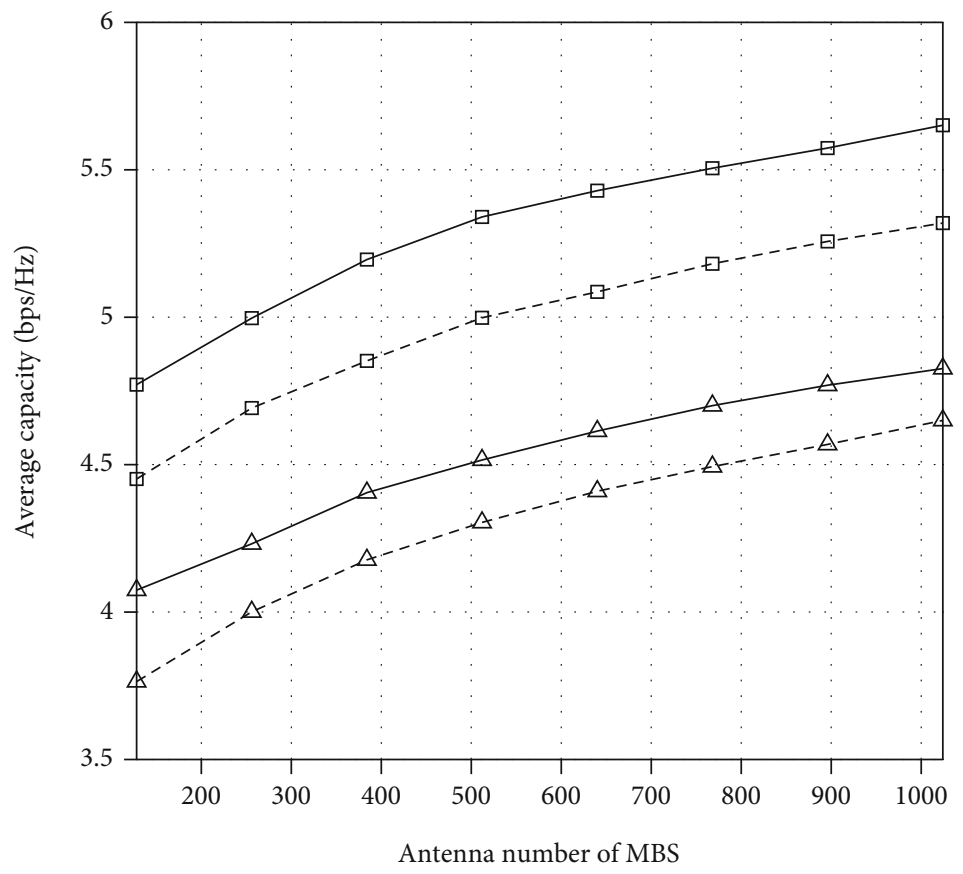

$$
\begin{aligned}
& \square \text { Proposed allocation (uniform AOA) } \\
& \triangle \text { Average allocation (uniform AOA) } \\
& -\boxminus-\text { Proposed allocation (Gaussian AOA) } \\
& -\triangle-\text { Average allocation (Gaussian AOA) }
\end{aligned}
$$

FIgURE 6: Average per SN uplink capacity versus antenna number of MBS.

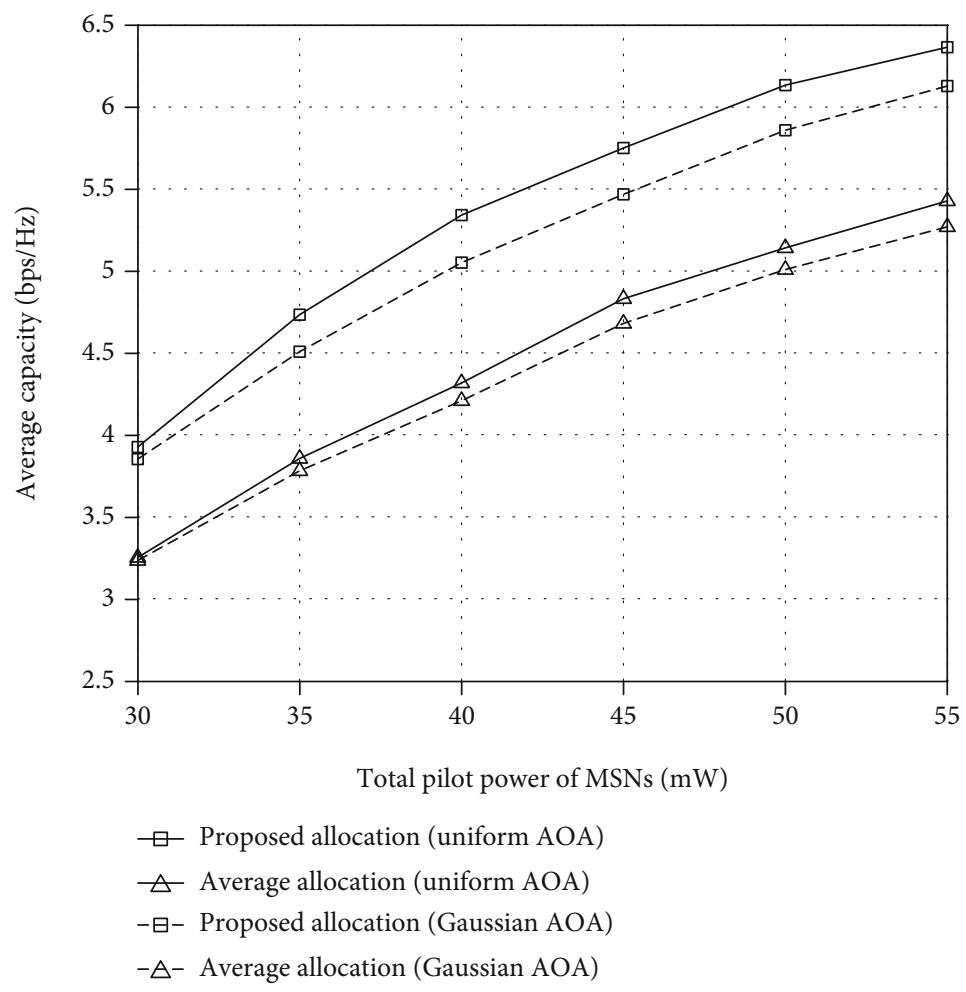

FIgURE 7: Average capacity versus the total pilot power of MSNs. 
In the simulation, we use the channel estimation which is proposed in the previous section, and AOAs have Gaussian and uniform distributions. We consider that AOAs have uniform distribution when $\theta \in\left[\bar{\theta}-\theta_{\Delta}, \bar{\theta}+\theta_{\Delta}\right]$, where $\bar{\theta}$ is the line of sight $\mathrm{AOA}$ and $\theta_{\Delta}=\delta_{\theta} / 2$. In another assumption that AOAs are Gaussian random variables, the mean and variance are $\bar{\theta}$ and $\left(\delta_{\theta} / 2\right)^{2}$, respectively, where $\delta_{\theta}$ is the angle spread.

The proposed pilot allocation mode can be evaluated using the average uplink capacity of each user. In Figure 6, the relationship between the average uplink capacity of each MSN and the number of MBSs' antennas in this scheme is shown, and the total pilot power is $40 \mathrm{~mW}$. In the figure, the "average allocation" scheme has poor performance. This is because it equally distributes the pilot transmit power to MSNs without any difference and does not take into account that different MSNs are affected by pilot-related pollution to different degrees. The "proposed allocation" scheme outperforms the "average allocation" scheme because it allocates more pilot transmit power to MSNs with better channel conditions. In two pilot power allocation modes, because some useful paths are filtered out and AOA obeys Gaussian distribution in an interval, so the Gaussian distribution of the AOA average uplink capacity is lower than the uniform distribution of AOA. With the increase of MBS antenna number, each under two kinds of AOA distribution MSN will significantly improve the average uplink transmission capacity. Different AOAs have higher resolutions, and pilot frequency reuse caused by interference will be greatly reduced.

Figure 7 shows the relationship of the average uplink capacity and the total pilot power of MSNs in two schemes, that is, pilot power average and distinguishing allocation. As the total transmitted power of the pilot increases, the average uplink capacity increases significantly. Moreover, regardless of the distribution of AOAs, with the increase of total power, the average power distribution obtained by the proposed power distribution scheme differs greatly from the uplink average capacity. The larger the $p_{\text {total }}$ value, the more transmitted power is allocated to the WeChat nodes with better channel gain, which tends to have a fundamental impact on system capacity.

\section{Conclusion}

In order to improve the uplink capacity of the MIMOHWSN system, a pilot transmission power scheme based on the traditional water-filling algorithm is proposed. Location-aware pilot allocation and channel estimation are introduced to suppress interference. When the total pilot transmitting power is fixed, based on the proposed pilot transmitting power scheme, more transmitting power can be allocated to a MSN with better channel gain, and the system capacity can be improved. Simulation results show that this method has the ability to significantly improve the uplink capacity when considering the average power allocation. These contributions will certainly improve the performance of $6 \mathrm{G}$ systems and make the quality of service of users much higher than $5 \mathrm{G}$ and bring some additional benefits.

\section{Data Availability}

The reader can contact us to get the paper data.

\section{Conflicts of Interest}

The authors declare that they have no conflicts of interest.

\section{References}

[1] Z. Chu, Z. Zhu, F. Zhou, M. Zhang, and N. Al-Dhahir, "Intelligent reflecting surface assisted wireless powered sensor networks for internet of things," IEEE Transactions on Communications, vol. 69, no. 7, pp. 4877-4889, 2021.

[2] S. Jacob, S. Joseph, P. G. Vinoj et al., "A novel spectrum sharing scheme using dynamic long short-term memory with CPOFDMA in 5G networks," IEEE Transactions on Cognitive Communications and Networking, vol. 6, no. 3, pp. 926-934, 2020.

[3] D. T. Do, T. T. T. Nguyen, C. B. le, M. Voznak, Z. Kaleem, and K. M. Rabie, "UAV relaying enabled NOMA network with hybrid duplexing and multiple antennas," IEEE Access, vol. 8, pp. 186993-187007, 2020.

[4] D. Zhang, Z. Zhou, S. Mumtaz, J. Rodriguez, and T. Sato, “One integrated energy efficiency proposal for $5 \mathrm{G}$ IoT communications," IEEE Internet of Things Journal, vol. 3, no. 6, pp. 1346-1354, 2016.

[5] X. Li, M. Zhao, Y. Liu, L. Li, Z. Ding, and A. Nallanathan, "Secrecy analysis of ambient backscatter NOMA systems under I/Q imbalance," IEEE Transactions on Vehicular Technology, vol. 69, no. 10, pp. 12286-12290, 2020.

[6] D. T. Do, M. S. Van Nguyen, T. N. Nguyen, X. Li, and K. Choi, "Enabling multiple power beacons for uplink of NOMAenabled mobile edge computing in wirelessly powered IoT," IEEE Access, vol. 8, pp. 148892-148905, 2020.

[7] Z. Chu, F. Zhou, Z. Zhu, R. Q. Hu, and P. Xiao, "Wireless powered sensor networks for internet of things: maximum throughput and optimal power allocation," IEEE Internet of Things Journal, vol. 5, no. 1, pp. 310-321, 2018.

[8] H. Niu, Z. Chu, F. Zhou, Z. Zhu, M. Zhang, and K. -K. Wong, "Weighted sum secrecy rate maximization using intelligent reflecting surface," IEEE Transactions on Communications, p. $1,2021$.

[9] X. Li, J. Li, Y. Liu, Z. Ding, and A. Nallanathan, "Residual transceiver hardware impairments on cooperative NOMA networks," IEEE Transactions on Wireless Communications, vol. 19, no. 1, pp. 680-695, 2020.

[10] T. L. Marzetta, "Noncooperative cellular wireless with unlimited numbers of base station antennas," IEEE Transactions on Wireless Communications, vol. 9, no. 11, pp. 3590-3600, 2010.

[11] Z. Zhu, Z. Chu, F. Zhou, H. Niu, Z. Wang, and I. Lee, "Secure beamforming designs for secrecy MIMO SWIPT systems," IEEE Wireless Communications Letters, vol. 7, no. 3, pp. 424427, 2018.

[12] Z. Zhu, Z. Chu, N. Wang, S. Huang, Z. Wang, and I. Lee, "Beamforming and power splitting designs for AN-aided secure multi-user MIMO SWIPT systems," IEEE Transactions on Information Forensics and Security, vol. 12, no. 12, pp. 2861-2874, 2017.

[13] S. Wang, J. Wan, D. Zhang, D. Li, and C. Zhang, "Towards smart factory for industry 4.0: a self-organized multi-agent 
system with big data based feedback and coordination," Computer Networks, vol. 101, pp. 158-168, 2016.

[14] X. Wu, H. Wang, D. Wei, and M. Shi, "ANFIS with natural language processing and gray relational analysis based cloud computing framework for real time energy efficient resource allocation," Computer Communications, vol. 150, pp. 122$130,2020$.

[15] X. Wu, Y. Wei, T. Jiang, Y. Wang, and S. Jiang, "A microaggregation algorithm based on density partition method for anonymizing biomedical data," Current Bioinformatics, vol. 14, no. 7, pp. 667-675, 2019.

[16] J. M. Williams, R. Khanna, J. P. Ruiz-Rosero et al., "Weaving the wireless Web: toward a low-power, dense wireless sensor network for the industrial IoT," IEEE Microwave Magazine, vol. 18, no. 7, pp. 40-63, 2017.

[17] Z. Zhu, S. Huang, Z. Chu, F. Zhou, D. Zhang, and I. Lee, "Robust designs of beamforming and power splitting for distributed antenna systems with wireless energy harvesting," IEEE Systems Journal, vol. 13, no. 1, pp. 30-41, 2019.

[18] Z. Zhu, N. Wang, W. Hao, Z. Wang, and I. Lee, "Robust beamforming designs in secure MIMO SWIPT IoT networks with a non-linear channel model," IEEE Internet of Things Journal, vol. 8, no. 3, pp. 1702-1715, 2021.

[19] I. Khan, F. Belqasmi, R. Glitho, N. Crespi, M. Morrow, and P. Polakos, "Wireless sensor network virtualization: a survey," IEEE Communications Surveys \& Tutorials, vol. 18, no. 1, pp. 553-576, 2016.

[20] M. Kountouris and N. Pappas, "HetNets and massive MIMO: modeling, potential gains, and performance analysis," in 2013 IEEE-APS Topical Conference on Antennas and Propagation in Wireless Communications (APWC), pp. 1319-1322, Turin, Italy, 2013.

[21] Z. Zhu, W. Ge, N. Wang et al., "AN-based beamforming design in secrecy heterogeneous WSN with MIMO-SWIPT," in IEEE international conference on communications workshops (ICC workshops), pp. 1-6, Shanghai, China, 2019.

[22] Z. Chu, F. Zhou, P. Xiao et al., "Resource allocation for secure wireless powered integrated multicast and unicast services with full duplex self-energy recycling," IEEE Transactions on Wireless Communications, vol. 18, no. 1, pp. 620-636, 2019.

[23] T. X. Vu, T. A. Vu, and T. Q. S. Quek, "Successive pilot contamination elimination in multiantenna multicell networks," IEEE Wireless Communications Letters, vol. 3, no. 6, pp. 617620, 2014.

[24] Y. Yao, Z. Zhu, S. Huang, X. Yue, C. Pan, and X. Li, "Energy efficiency characterization in heterogeneous IoT system with UAV swarms based on wireless power transfer," IEEE Access, vol. 8, pp. 967-979, 2020.

[25] J. K. Tugnait, "Self-contamination for detection of pilot contamination attack in multiple antenna systems," IEEE Wireless Communications Letters, vol. 4, no. 5, pp. 525-528, 2015.

[26] A. Khansefid and H. Minn, "On channel estimation for massive MIMO with pilot contamination," IEEE Communications Letters, vol. 19, no. 9, pp. 1660-1663, 2015.

[27] J.-C. Shen, J. Zhang, and K. B. Letaief, "Downlink user capacity of massive MIMO under pilot contamination," IEEE Transactions on Wireless Communications, vol. 14, no. 6, pp. 31833193, 2015.

[28] O. Elijah, C. Y. Leow, T. A. Rahman, S. Nunoo, and S. Z. Iliya, "A comprehensive survey of pilot contamination in massive
MIMO-5G system," IEEE Communications Surveys Tutorials, vol. 18, no. 2, pp. 905-923, 2016.

[29] I. E. Shaalan, A. A. Khattaby, and A. S. Dessouki, “A new joint TSPA/WGC pilot contamination reduction strategy based on exact graph coloring grouping algorithm," IEEE Access, vol. 7, pp. 150552-150564, 2019.

[30] J. Jose, A. Ashikhmin, T. L. Marzetta, and S. Vishwanath, "Pilot contamination problem in multi-cell TDD systems," in 2009 IEEE International Symposium on Information Theory, pp. 2184-2188, Seoul, Korea (South), 2009.

[31] Z. Wang, P. Zhao, C. Qian, and S. Chen, "Location-aware channel estimation enhanced TDD based massive MIMO," IEEE Access, vol. 4, no. 99, pp. 7828-7840, 2016.

[32] T. Lian, L. You, W. Zhong, and X. Gao, "Coordinated pilot reuse for multi-cell massive MIMO transmission," in International Symposium on Personal, Indoor and Mobile Radio Communications, pp. 492-496, Washington, DC, USA, 2014.

[33] H. Yin, D. Gesbert, M. Filippou, and Y. Liu, "A coordinated approach to channel estimation in large-scale multipleantenna systems," IEEE Journal on Selected Areas in Communications, vol. 31, no. 2, pp. 264-273, 2013.

[34] R. R. Muller, L. Cottatellucci, and M. Vehkapera, "Blind pilot decontamination," IEEE Journal of Selected Topics in Signal Processing, vol. 8, no. 5, pp. 773-786, 2014.

[35] H. Q. Ngo and E. G. Larsson, "EVD-based channel estimation in multicell multiuser MIMO systems with very large antenna arrays," in International Conference on Acoustics, Speech and Signal Processing, pp. 3249-3252, Kyoto, Japan, 2012.

[36] L. You, X. Gao, X. G. Xia, N. Ma, and Y. Peng, "Pilot reuse for massive MIMO transmission over spatially correlated Rayleigh fading channels," IEEE Transactions on Wireless Communications, vol. 14, no. 6, pp. 3352-3366, 2015.

[37] L. Su and C. Yang, "Fractional frequency reuse aided pilot decontamination for massive MIMO systems," in 81st Vehicular Technology Conference, IEEE, pp. 1-6, Glasgow, UK, 2015.

[38] I. Atzeni, J. Arnau, and M. Debbah, "Fractional pilot reuse in massive MIMO systems," in International Conference on Communication Workshop, pp. 1030-1035, London, UK, 2015.

[39] X. Zhu, Z. Wang, C. Qian et al., "Soft pilot reuse and multi-cell block diagonalization precoding for massive MIMO systems," IEEE Transactions on Vehicular Technology, vol. 65, no. 5, pp. 3285-3298, 2016.

[40] J.-C. Chen, C.-K. Wen, S. Jin, and K.-K. Wong, "A low complexity pilot scheduling algorithm for massive MIMO," IEEE Wireless Communications Letters, vol. 6, no. 1, pp. 18-21, 2017.

[41] Z. Chen and C. Yang, "Pilot decontamination in massive MIMO systems: exploiting channel sparsity with pilot assignment," in IEEE Global Conference on Signal and Information Processing, pp. 637-641, Atlanta, GA, USA, 2014.

[42] Z. Zhao, Z. Chen, and Y. Liu, "Cell sectorization-based pilot assignment scheme in massive MIMO systems," in IEEE Wireless Telecommunications Symposium, pp. 1-5, New York, NY, USA, 2015.

[43] M. Liu, X. Chen, W. Xu, H. Zhang, X. You, and W. Miao, "Grouped pilot reuse for channel estimation in massive MIMO networks," in IEEE International Conference on Wireless Communications and Signal Processing, pp. 1-5, Yangzhou, China, 2016.

[44] N. Akbar, S. Yan, N. Yang, and J. Yuan, "Mitigating pilot contamination through location-aware pilot assignment in 
massive MIMO networks," in IEEE Global Communications Conference Workshop, pp. 1-6, Washington, DC, USA, 2017.

[45] V. Saxena, G. Fodor, and E. Karipidis, "Mitigating pilot contamination by pilot reuse and power control schemes for massive MIMO systems," in IEEE 81t Vehicular Technology Conference, pp. 1-6, Glasgow, UK, 2015.

[46] T. M. Nguyen and L. B. Le, "Joint pilot assignment and resource allocation in multicell massive MlMO network: throughput and energy efficiency maximization," in IEEE Wireless Communications and Networking Conference, pp. 393-398, New Orleans, LA, USA, 2015. 